\title{
Energy Absorption of Seated Occupants Exposed to Horizontal Vibration and Role of Back Support Condition
}

\author{
Subhash RAKHEJA ${ }^{1 *}$, Santosh MANDAPURAM ${ }^{1}$ and Ren G. DONG ${ }^{2}$ \\ ${ }^{1}$ ConCAVE Research Centre, Mechanical \& Industrial Engineering, Concordia University, 1455 de \\ Maisonneuve West, Montreal, Quebec H3G 1M8, Canada \\ ${ }^{2}$ Engineering \& Control Technology Branch, National Institute for Occupational Safety and Health, 1095 \\ Willowdale Road, Morgantown, West Virginia 26505, USA
}

Received November 27, 2007 and accepted May 30, 2008

\begin{abstract}
Absorbed power characteristics of seated human subjects under fore-aft ( $x$-axis) and lateral (y-axis) vibration are investigated through measurements of dynamic interactions at the two driving-points formed by the body and the seat pan, and upper body and the backrest. The experiments involved: (i) three back support conditions (no back support, and back supported against a vertical and an inclined backrest); (ii) three seat pan heights $(425,390$ and $350 \mathrm{~mm}$ ); and three magnitudes $\left(0.25,0.5\right.$ and $1.0 \mathrm{~m} / \mathrm{s}^{2} \mathrm{rms}$ acceleration) of band limited random excitations in $0.5-10 \mathrm{~Hz}$ frequency range, applied independently along the $x$ - and $y$ - axes. The force responses, measured at the seat pan and the backrest are applied to characterize total energy transfer reflected on the seat pan and the backrest. The mean responses suggest strong contributions due to back support, and direction and magnitude of vibration. In the absence of a back support, the seat pan responses dominated in lower frequency bands centered at 0.63 and $1.25 \mathrm{~Hz}$ under both directions of motion. Most significant interactions of the upper body against the back support was observed under fore-aft vibration. The addition of back support caused the seat pan response to converge to a single primary peak near a higher frequency of $4 \mathrm{~Hz}$ under $x$ axis, with only little effect on the $y$-axis responses. The back support serves as an additional source of vibration to the occupant and an important constraint to limit the fore-aft movement of the upper body and thus relatively higher energy transfer under. The mean responses were further explored to examine the $W_{d}$ frequency-weighting used for assessing exposure to horizontal vibration. The results show that the current weighting is suited for assessing the vibration exposure of human subjects seated only without a back support.
\end{abstract}

Key words: Absorbed power, Horizontal vibration, Back support condition, Seat height, Body-seat interactions, Upper body-backrest interactions

\section{Introduction}

Occupational off road vehicle drivers are exposed to considerable magnitudes of whole-body vibration (WBV),

\footnotetext{
*To whom correspondence should be addressed.

Disclaimers: The content of this publication does not necessarily reflect the views or policies of the National Institute for Occupational Safety and Health (NIOSH), nor does mention of trade names, commercial products, or organizations imply endorsement by the U.S. Government.
}

which is known to cause discomfort, annoyance, and several health and safety risks. Many studies have suggested strong association between the exposure to WBV and low back pain ${ }^{1,2)}$. The vast majority of the studies on human responses to vibration have emphasized the exposure to vertical WBV, since heavy on-road and off-road vehicles are believed to transmit relatively higher magnitudes of vertical vibration than those along the other axes. Such vehicles, however, also transmit substantial magnitudes of horizontal vibration (HV) along the fore-aft and 
Table 1. Magnitudes of frequency weighted rms accelerations due to vibration measured along the $x$-, $y$ - and $z$ - axis on the seats of the heavy vehicles ${ }^{3-7)}$

\begin{tabular}{llll}
\hline Vehicle & $a w_{x}\left(\mathrm{~m} / \mathrm{s}^{2}\right)$ & $a w_{y}\left(\mathrm{~m} / \mathrm{s}^{2}\right)$ & $a w_{z}\left(\mathrm{~m} / \mathrm{s}^{2}\right)$ \\
\hline Tracked forestry vehicle & $\approx 0.25$ & $\approx 0.12$ & $\approx 0.39$ \\
Cargo trucks (1-2 Tons) & $0.36-0.70$ & $0.39-0.75$ & $0.65-1.29$ \\
Cargo trucks (> 10 Tons) & $0.20-0.42$ & $0.20-0.24$ & $0.42-0.70$ \\
All terrain vehicles (cargo) & $0.30-1.0$ & $0.50-1.10$ & $1.0-1.80$ \\
On-road passenger vehicle & $0.17-0.23$ & $0.38-0.54$ & $0.59-0.62$ \\
(rough surface) & & & \\
Mini city bus & $0.10-0.60$ & $0.00-0.90$ & $0.20-0.60$ \\
Fork lift (off-road) & $0.10-0.90$ & $0.10-2.50$ & $0.50-1.60$ \\
Port crane & $0.80-1.30$ & $\approx 0.10$ & $\approx 0.10$ \\
Dump truck 2 Ton & $0.29-1.31$ & $0.23-1.72$ & $0.30-1.64$ \\
Garbage 4 Ton & $0.50-0.94$ & $0.56-1.98$ & $0.37-2.45$ \\
\hline
\end{tabular}

side-to-side axes ${ }^{3-7)}$. Table 1 lists examples of the frequency-weighted rms accelerations $\left(a_{w x}, a_{w y}\right.$ and $\left.a_{w z}\right)$ due to vibration transmitted along the fore-aft $(x$-), side-to-side or lateral $(y-)$ and vertical $(z-)$ axes of various industrial and heavy road vehicles, derived on the basis of $W_{d^{-}}$and $W_{k^{-}}$weighting filters defined in ISO 2631-1 ${ }^{8)}$. These data suggest that drivers of such vehicles are also exposed to considerable magnitudes of weighted $\mathrm{HV}$, which may even approach or exceed the magnitudes of vertical vibration in some of the vehicles.

Despite the substantial magnitudes of HV, relatively fewer studies have investigated the seated body response to HV. Furthermore, the majority of the reported studies on HV have focused on the motion sickness (kinetosis) response under extremely low frequency vibration $(\leq 1 \mathrm{~Hz})$. The motion sickness caused by low frequency HV is known to impede an operator's ability to handle the vehicle and perform desired tasks, while the symptoms have been characterised as temporary minor annoyances in most of the cases ${ }^{9)}$. Considerable efforts have been made to characterise seated human biodynamical response to vibration and contributing factors, in terms of forcemotion relationships, such as apparent mass (APMS) and driving-point mechanical impedance (DPMI) ${ }^{10-12)}$. These studies have provided the resonance frequencies and guidance to the sensitivity of seated human body to WBV. The vast majority of the reported studies have concentrated on vertical vibration; only a few have investigated the biodynamic response to $\mathrm{HV}^{12-16)}$.

The acceleration due to source vibration measured at either the seat or the floor, on the other hand, is considered to represent the vibration hazard ${ }^{8}$. The vibration hazard takes effect through the biodynamic response of the human body ${ }^{17)}$. The vibration power absorbed $\left(P_{A b s}\right)$ by the exposed body is a measure that combines both the vibration hazard and the biodynamic response of the body.
Physically, the absorbed power relates to dissipation of energy attributed to relative motions of the visco-elastic tissues, muscles and skeletal system, which under prolonged exposures could lead to physical damages in the musculoskeletal system ${ }^{18,19)}$. Mathematically, the absorbed power can be computed from the integration of the power absorption density, which is equivalent of the product of vibration-induced stress and the strain rate, over the volume of tissues involved in the biodynamic response, which includes the essential mechanical stimuli that cause the biological responses and adaptation ${ }^{17)}$. It is thus reasonable to hypothesize that the power absorption is associated with the vibration-induced discomfort and some health effects. However, their exact relationship has not been sufficiently studied.

The concept of energy absorbed by the seated human body exposed to seat-transmitted vibration, first evolved in the mid-60's as a measure for evaluating the safety and comfort of occupants of military vehicles ${ }^{18)}$. The absorbed power responses of the seated human body exposed to vertical WBV, have been investigated under continuous sinusoidal and random vibration considering both supported and unsupported back postures ${ }^{20-25}$. The $P_{A b s}$ has been related to APMS and DPMI ${ }^{26)}$, and the reported $P_{A b s}$ spectra generally exhibit peaks at frequencies that are comparable to those corresponding to APMS/DPMI magnitude peaks. The $P_{A b s}$ response of the body increases nearly quadratically with the acceleration magnitude. The studies have also shown important influences of variations in the sitting posture and seat geometry factors (seat height, footrest position, hands position, backrest and seat pan angle) on the $P_{A b s}$ response of human occupants exposed to vertical $\mathrm{WBV}^{21,22)}$. Relatively larger magnitudes of absorbed power have been associated with mechanical shock stimuli compared to the continuous vibration, suggesting greater sensitivity of the human body response to shocks ${ }^{20}$.

Unlike the vertical WBV, the $P_{A b s}$ responses of seated subjects under $\mathrm{HV}$ have been reported only from one study. Lundstrom, et al. ${ }^{23)}$ reported the $P_{A b s}$ characteristics of seated male and female subjects, exposed to $x$ - and $y$-axis sinusoidal vibration at various discrete frequencies in the 1.13 to $80 \mathrm{~Hz}$ range. The experiments were performed with subjects seated without a back support with feet on a stationary support and exposed to different magnitudes of vibration ( $\mathrm{rms}$ acceleration ranging from 0.25 to $1.4 \mathrm{~m} / \mathrm{s}^{2}$ ). The measured data under $x$ - and $y$ - axes vibration revealed dominant energy dissipation at frequencies below $3 \mathrm{~Hz}$, while considerably large inter-subject variability was observed at frequencies up to $10 \mathrm{~Hz}$. The study also reported that the $W_{d^{-}}$frequency weighting, defined in ISO $2631-1^{8)}$, underestimates the exposure risk in the $1.5-3 \mathrm{~Hz}$ frequency range, and overestimates 
the risk at frequencies above $5 \mathrm{~Hz}$. The study also suggested need for differential guidelines assessing HV exposures risks for females and males.

The biodynamic responses of the seated body exposed to WBV are known to depend upon back support condition and posture in a highly complex manner. Moreover, the body-seat system represents multiple driving-points formed by the lower body-seat pan, upper body-backrest, hands-steering wheel and feet-footrest interfaces. A single driving-point formed by the lower body and seat pan, however, has been mostly considered in the reported biodynamic studies, irrespective of the axis of WBV. Nawayseh and Griffin ${ }^{27)}$ and Rakheja et al. ${ }^{28)}$ performed measurements of biodynamic responses of the seated body at the seat pan and back support interfaces under vertical vibration using a vertical and an inclined backrest, respectively; which were reported in terms of forces at the backrest and cross-axes APMS. These studies considered negligible contributions due to driving-points formed by the feet and the hands. These studies revealed significant dynamic interactions of the upper body with the backrest; the magnitude of the biodynamic force measured at the inclined backrest along a direction normal to the back support was substantial even though the vibration was applied along the vertical axis.

The seated body is expected to exhibit greater interactions with the backrest under fore-aft HV, which have not been adequately quantified. The characterisation of biodynamic responses to $\mathrm{HV}$ thus necessitates consideration of at least two important driving-points formed by the lower body-pan and upper body-backrest interfaces. The biodynamic responses in terms of APMS/DPMI of the seated body to HV have been mostly measured at the body-seat pan interface with either no back support $^{12,14,15,29)}$ or a vertical back support ${ }^{14,15)}$. The forces developed at a vertical back support and the APMS under HV have been reported in a recent study by Nawayseh and Griffin ${ }^{12)}$. Mandapuram et al. ${ }^{14)}$ reported the APMS responses for both vertical and an inclined backrest under fore-aft and side-to-side vibration. These studies revealed significant magnitudes of APMS response measured at the backrest, when compared to that measured at the seat pan.

The influence of back support condition on the dissipated energy under HV has not yet been reported. Moreover, a study of seated body interactions with the backrest, which also serves as a source of vibration excitation under HV, has not been attempted. In this study, the absorbed power characteristics of seated human subjects are investigated under fore-aft and side-to-side vibration at two driving-points formed by seated body-seat pan and the upper body-seat backrest interfaces. The experiments involved three different back support conditions, three different seat pan heights and three different magnitudes of band limited random excitations in the 0.5-10 $\mathrm{Hz}$ frequency range, applied independently along the $x$ and $y$-axes. The biodynamic force responses, measured at the seat pan and the backrest along the direction of motion, are applied to characterize the total body $P_{A b s}$ reflected on the seat pan, and that of the upper body reflected on the backrest.

\section{Methods}

A rigid seat with adjustable backrest and height was designed for the experiments. The seat consisted of a $500 \times 400 \mathrm{~mm}$ flat seat pan and a $470 \mathrm{~mm}$ high backrest installed on a truss structure. The seat was installed on a HV simulator through two three-axis force plates (Kistler 9257AB each $170 \times 140 \mathrm{~mm}$ ) capable of measuring forces at the seat base along the three translational axes. A summing junction was used to sum the force signals from the two force plates along the respective axes to compute the resultant dynamic force due to the rigid seat and the occupant at the seat pan interface, as illustrated in the $x$-z plane of the seat in Fig. 1, for the $x$-axis motion. An additional three-axis force plate (Kistler $9257 \mathrm{~A}, 170 \times 140 \mathrm{~mm}$ ) was also installed between the backrest support plate and the seat back truss structure to capture the dynamic forces arising at the occupant's upper body and the backrest interface. Under $x$-axis motion, the force acting along an axis normal to the backrest alone, however, was acquired, since the forces along the sideto-side (lateral) and vertical directions of the backrest were expected to be significantly small in magnitude ${ }^{13}$. Under $y$ - axis excitations, the measurement of backrest force was limited to $y$-axis alone. A pictorial view of the test seat installed on the vibration platform for measurement of responses under $y$-axis excitation is illustrated in Fig. 2. Single-axis accelerometers (Analog Devices, ADXL) were further installed on the seat back and the platform, oriented along the axis of the motion, to capture the acceleration due to excitations at the two drivingpoints. The seat was designed such that it could be easily oriented along the $x$ - or $y$-axis of motion of the vibration platform, which consisted of a magnesium slip table sliding on an oil film over a granite slab. The slip table was driven by a $48 \mathrm{~cm}$ stroke servo-controlled hydraulic actuator.

The experiments were performed under excitations along the $x$ - and $y$-axes, applied in an uncoupled manner. A total of 8 healthy male volunteers, aged between 21-51 yr, took part in the experiment. The subjects had no prior known history of musculo-skeletal system disorders. The subjects' mass ranged from $59.4 \mathrm{~kg}$ to $92 \mathrm{~kg}$, with mean mass of $71.2 \mathrm{~kg}$ and standard deviation of the 

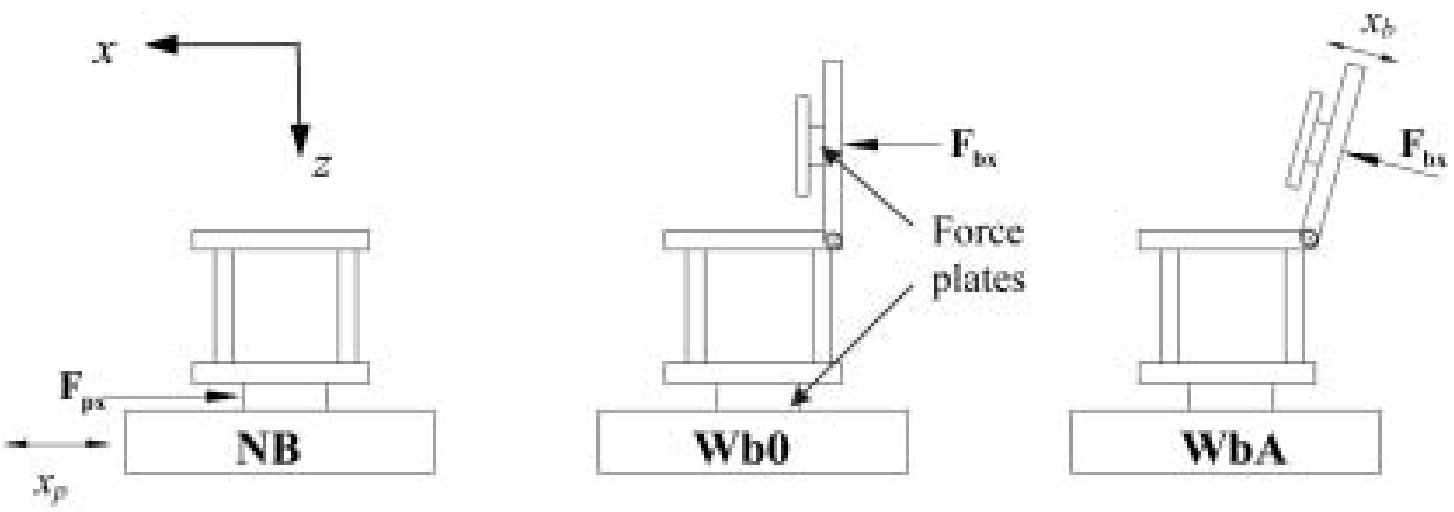

Fig. 1. Schematic illustrations of the three different sitting postures used in the study under fore-aft ( $x$-axis) vibration. (NB - No back support; Wb0 - Vertical back support; WbA - Inclined back support).

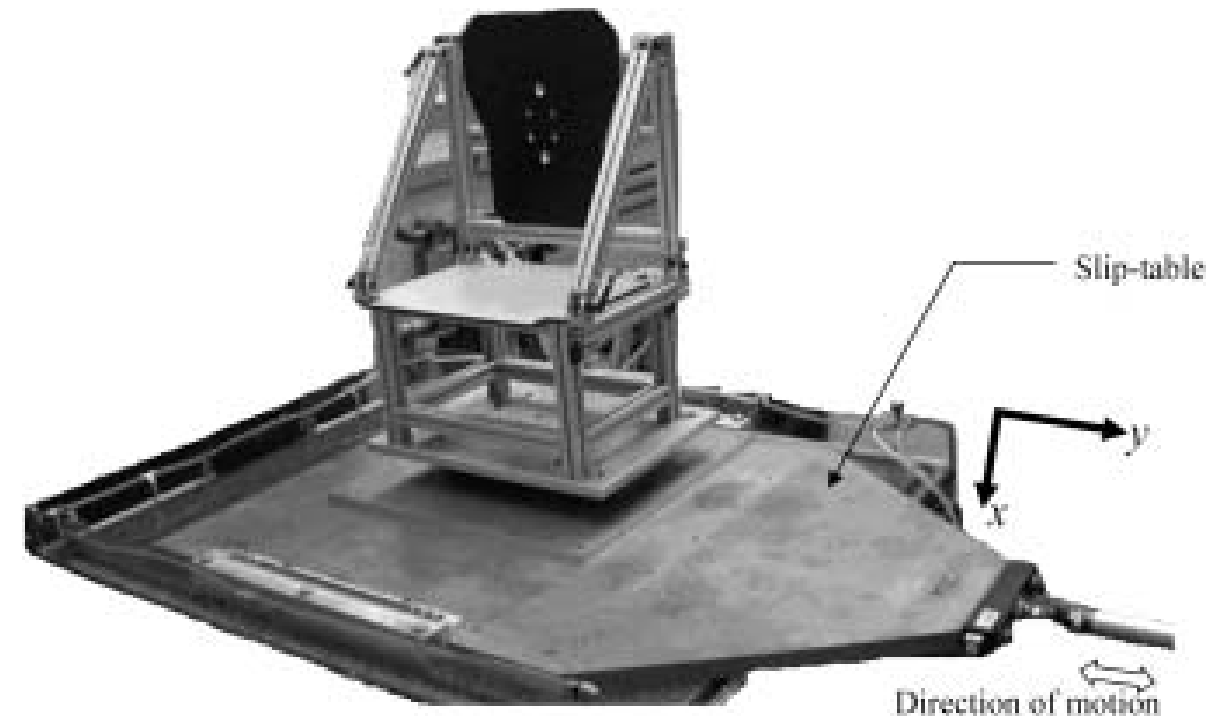

Fig. 2. Arrangement of the test seat on the horizontal vibration platform for measurement of the responses under $y$-axis excitation.

mean of $10.6 \mathrm{~kg}$. The standing height of the subjects varied from $1.70 \mathrm{~m}$ to $1.78 \mathrm{~m}$ (mean $=1.73 \mathrm{~m}$; standard deviation $=0.025 \mathrm{~m}$ ). Prior to the test, each subject was informed about the purpose of the study, experimental set up and usage of the hand-held emergency stop. Subjects were given written information about the experiment and were requested to sign a consent form that was previously approved by a Human Research Ethics Committee.

The measurements were performed for each subject assuming different sitting postures realized by three different back support conditions and three different seat heights (referred to as $\mathrm{H}_{1}, \mathrm{H}_{2}$ and $\mathrm{H}_{3}$ ). The back support conditions included: (i) sitting erect with no back support, NB; (ii) Sitting erect with upper body supported against a vertical backrest, $\mathrm{Wb0}$; and (iii) seated relaxed with upper body supported against backrest inclined at an angle of $12.5^{\circ}$ with respect to the vertical axis WbA (Fig. 1).
The measurements of energy absorption were performed under three different levels of constant acceleration spectral density random excitations in the $0.5-10 \mathrm{~Hz}$ frequency range applied independently along the $x$ - and $y$ axes. The overall rms accelerations of the selected excitations for the two directions were $0.25,0.5$ and $1.0 \mathrm{~m} / \mathrm{s}^{2}$. The subjects were seated with their hands in the lap and feet supported on the moving platform for each posture. The vibration transmitted from the feet to the upper body is assumed to be small relative to the source vibration at the pan and the back support. Each subject wore a cotton lab coat to ensure uniform friction between the back and the backrest, which was judged to be an important factor under lateral excitation. Each test was performed two times and the data were examined for repeatability. The resulting test matrix involved a total of 54 trials for each axis of vibration, comprising three types of back 
Table 2. Test matrix

\begin{tabular}{llll}
\hline Posture & No-back support, NB & Vertical back support, Wb0 & Inclined back support, WbA \\
Excitation Magnitude $(\mathrm{rms})$ & $0.25 \mathrm{~m} / \mathrm{s}^{2}$ & $0.5 \mathrm{~m} / \mathrm{s}^{2}$ & $1.0 \mathrm{~m} / \mathrm{s}^{2}$ \\
Seat height & $\mathrm{H}_{1}(425 \mathrm{~mm})$ & $\mathrm{H}_{2}(390 \mathrm{~mm})$ & $\mathrm{H}_{3}(350 \mathrm{~mm})$ \\
Direction of motion & Fore-aft $(x)$ & Lateral $(y)$ & \\
\hline
\end{tabular}

support conditions, three seat heights, three excitation levels and two repeats. Table 2 summarizes the test matrix. The duration of each measurement was $128 \mathrm{~s}$, while the subject's posture during a trial was visually checked by the experimenter to ensure consistency. The experiments were randomized, and each participant was asked to dismount the seat and vibration platform after each trial to relax for at least $2 \mathrm{~min}$.

\section{Analysis of Absorbed Power}

The instantaneous power $P(t)$ absorbed by the human body can be computed from the force exerted on the body $F(t)$ at the body-seat interface and the velocity $v(t)$ due to vibration excitation:

$P(t)=F(t) \cdot v(t)$

The average vibration energy transferred to the body during a period $\mathrm{T}$ can be expressed as:

$$
P_{(\text {avg })}=\int_{t_{0}}^{t_{0}+T} F(t) v(t) d t
$$

The absorbed power in the frequency domain can be obtained from the cross-spectrum of the force and velocity, such that ${ }^{18)}$ :

$P(j \omega)=S_{F v}(j \omega)=C(\omega)-j Q(\omega)$

Where $S_{F v}(j \omega)$ is the complex co-spectrum of measured force and velocity, $C(\omega)$ is the coincident spectral density function (co-spectrum) and imaginary component $Q(\omega)$ is referred to as quadrature spectral density function (quad-spectrum). In the context of the vibration energy transferred to a seated human body, the real component reflects the energy dissipated in the biological structure per unit time and the imaginary component reflects the energy stored/released by the system ${ }^{23)}$. The biological system with finite damping consumes the vibratory energy by means of relative motions between the tissues, muscles and skeletal systems, which is transformed into heat. It has been speculated that this dissipative component could be related to musculoskeletal disorders, while the restoring part relates to vibration comfort and perception.

The vibration power absorbed by the vibration-exposed seated body $P_{a}(\omega)$ is thus expressed as the real part of the cross-spectrum between the force and velocity signals, such that:

$P_{a}(\omega)=\operatorname{Re}\left[S_{F v}(j \omega)\right]$

Where Re designates the real component.

The absorbed power can also be evaluated from the apparent mass using an indirect apparoach ${ }^{22,26)}$ :

$$
P_{a}(\omega)=\int_{0}^{\infty} \frac{\operatorname{Im}\left[\left(M^{*}(\omega)\right)\right] S_{a a}(\omega)}{\omega} d \omega
$$

Where $M^{*}$ is the complex conjugate of the apparent mass, ' $I m$ ' designates the imaginary part and $S_{a a}$ is the spectral density of the acceleration excitation.

In this study, instantaneous forces developed at the base and the backrest were acquired together with the acceleration signals in the multi-channel Pulse Labshop ${ }^{\mathrm{TM}}$. The data were analyzed to compute the absorbed power responses at the seat base and the backrest attributed to the forces measured at the two driving-points, respectively. Under the $x$-axis vibration, the absorbed power responses were computed from the forces measured at the two driving-points, such that:

$P_{p x}(\omega)=\operatorname{Re}\left[S_{F_{p x} v_{p x}}\right] ;$ and $P_{b x}(\omega)=\operatorname{Re}\left[S_{F_{b x} v_{b x}}\right]$

Where $P_{p x}$ and $P_{b x}$ are the absorbed power responses measured at the seat pan and the backrest, respectively, under $x$ - axis vibration. $F_{p x}$ and $F_{b x}$ are the respective forces measured at the seat pan and the backrest. $v_{p x}$ and $v_{b x}$ are velocities measured at the seat pan and the backrest, respectively, due to $x$ - axis vibration. Owing to the rigid nature of the seat structure the two velocities were found to be identical $\left(v_{p x}=v_{b x}\right)$ for the vertical backrest. $S_{F_{p x} v_{p x}}$ and $S_{F_{b x} v_{b x}}$ are the cross-spectra of the forces and velocities, at the pan and the backrest respectively. The absorbed power responses measured at the seat pan $\left(P_{p y}\right)$ and the backrest $\left(P_{b y}\right)$ under $y$-axis excitations were also evaluated in the similar manner.

Each subject was seated assuming the desired posture with hands on the lap and feet on the vibrating slip table. The selected excitation signal was then applied and the total forces measured at the seat pan and backrest were acquired to compute the $P_{A b s}$ responses of the seated occupant using Eq. (6) for the respective axis of vibration. The cross-spectra were obtained in the Pulse Labshop $^{\mathrm{TM}}$ using a band width of $50 \mathrm{~Hz}$ with frequency resolution of $0.0625 \mathrm{~Hz}$ and $75 \%$ overlap. The mea- 
surements were initially performed with the rigid seat alone. The data analysis resulted in negligible magnitude of absorbed power in the entire frequency range.

The coherence of the measured forces and accelerations were also evaluated and monitored during each trial. The measurements at the seat base invariably revealed high coherency of the force and acceleration signals under both axes of motion $(\geq 0.95)$ in the $0.5-10 \mathrm{~Hz}$ frequency range, irrespective of the sitting posture and the excitation level considered. The measurements at the backrest along $x$ and $y$ - axes also revealed coherence values greater than 0.95 under lower magnitude $\left(0.25 \mathrm{~m} / \mathrm{s}^{2}\right)$ of vibration for both back supported conditions ( $\mathrm{Wb} 0$ and $\mathrm{WbA}$ ). The coherence value decreased to 0.9 in the $0.5-4.5 \mathrm{~Hz}$ frequency range under higher magnitude of vibration $\left(1 \mathrm{~m} / \mathrm{s}^{2}\right)$ for both axes of motion. The coherency of the $y$-axis measurements increased with frequency in the higher frequency range for the $\mathrm{Wb} 0$ posture but decreased slightly for the $x$-axis measurements. This was most likely attributed to the pitching and rocking motions of the upper body, and intermittent loss of contact with the vertical backrest under fore-aft vibration. The measurements with the inclined backrest, however, revealed good coherency of the force and acceleration signals measured at the backrest under both axes of motion in the entire frequency range. The coherence values for the back supported conditions generally improved with the decrease in seat height suggesting increased stability and greater adhesion of the body with the supports.

The total absorbed power response of the human body subjected to either $x$ - or $y$ - axis vibration can be computed from integration of the real component of the crossspectrum density over the frequency range of interest. For $x$ - axis excitation, the total power may be derived from:

$$
\overline{P_{p x}}=\int_{\omega_{1}}^{\omega_{2}} \operatorname{Re}\left[S_{F_{p x} v_{p x}}(j \omega)\right] d \omega ; \text { and } \overline{P_{b x}}=\int_{\omega_{1}}^{\omega_{2}} \operatorname{Re}\left[S_{F_{b x} v_{b x}}(j \omega)\right] d \omega
$$

Where $\overline{P_{p x}}$ and $\overline{P_{b x}}$ are the total absorbed power responses measured at the seat pan and the backrest interfaces, respectively, under $x$-axis vibration. The limiting frequencies $\omega_{1}$ and $\omega_{2}$ define the frequency range of interest.

Alternatively, the total power may be derived upon summation of absorbed power responses corresponding to each one third-octave frequency band, such that:

$$
\overline{P_{p x}}=\sum_{i=1}^{N} P_{p x}\left(f_{i}\right) ; \text { and } \overline{P_{b x}}=\sum_{i=1}^{N} P_{b x}\left(f_{i}\right)
$$

Where $P_{p x}\left(f_{i}\right)$ and $P_{b x}\left(f_{i}\right)$ are the absorbed power responses at the centre frequency $f_{i}$ of the $i^{\text {th }} 1 / 3$-octave frequency band and $\mathrm{N}$ is the total number of frequency bands in the frequency range of interest.

The measured absorbed power responses of the seated subjects exposed to vibration generally show considerable variations. A number of studies on vertical biodynamic responses in terms of APMS of seated individuals and a few on HV biodynamics have mostly attributed the dispersion in the measured data to variations in the body mass. The APMS magnitude normalization with respect to the magnitude near $0.5 \mathrm{~Hz}$ or static seated mass have been widely employed to reduce the variability in vertical APMS data ${ }^{22-24)}$. The normalization factors for the seat pan and backrest APMS magnitudes under HV, were suggested as $87.8 \%$ and $67.8 \%$, respectively, of the total body mass ${ }^{14)}$, which represented portions of the body mass reflected at the seat pan and the backrest ${ }^{30}$. In a similar manner, different forms of normalized power have also been reported, such as power normalized by the body mass (W/kg), power density normalized by acceleration spectral density $\left(\mathrm{Ns}^{3} / \mathrm{m}\right)$ and that by the product of acceleration spectral density and the body mass $\left(\mathrm{Ns}^{3} / \mathrm{m} / \mathrm{kg}\right)$. It has been suggested that the normalization with respect to acceleration spectrum helps to smoothen the small magnitude oscillations in the power response ${ }^{24)}$. Lundström and Holmlund ${ }^{23}$ ) normalized the measured absorbed power spectra under HV with respect to the body mass supported by the seat pan in order to reduce the degree of dispersion in the data.

\section{Results and Discussions}

The averages of the measured absorbed power at the seat pan and the backrest of an individual in two trials were taken to derive the mean responses of each participant, as different trials revealed high degree of consistency. The mean $P_{A b s}$ responses obtained for the 8 subjects were evaluated at each of the one third-octave band centre frequency in the $0.5-10 \mathrm{~Hz}$ frequency range. Figure 3 illustrates the mean responses of 8 individuals measured at the seat pan under $1 \mathrm{~m} / \mathrm{s}^{2}$ excitation along the $x$ - and $y$ - axes $\left(P_{p x}\right.$ and $\left.P_{p y}\right)$. The figure shows responses measured with all three sitting conditions (NB, $\mathrm{Wb} 0$ and $\mathrm{WbA})$, while the seat height was $425 \mathrm{~mm}\left(\mathrm{H}_{1}\right)$. The results clearly show considerable variability in the measured absorbed power, although consistent trends with respect to spectral components could be observed for the three postures and two excitation directions considered. The results particularly show the concentration of magnitude peaks around the comparable frequency bands for most of the subjects. The NB posture generally caused a sensation of instability among the subjects, particularly under $x$-axis vibration, and encouraged the subjects to shift more weight to and from their feet, specifically when the displacement was perceived to be high. A consider- 

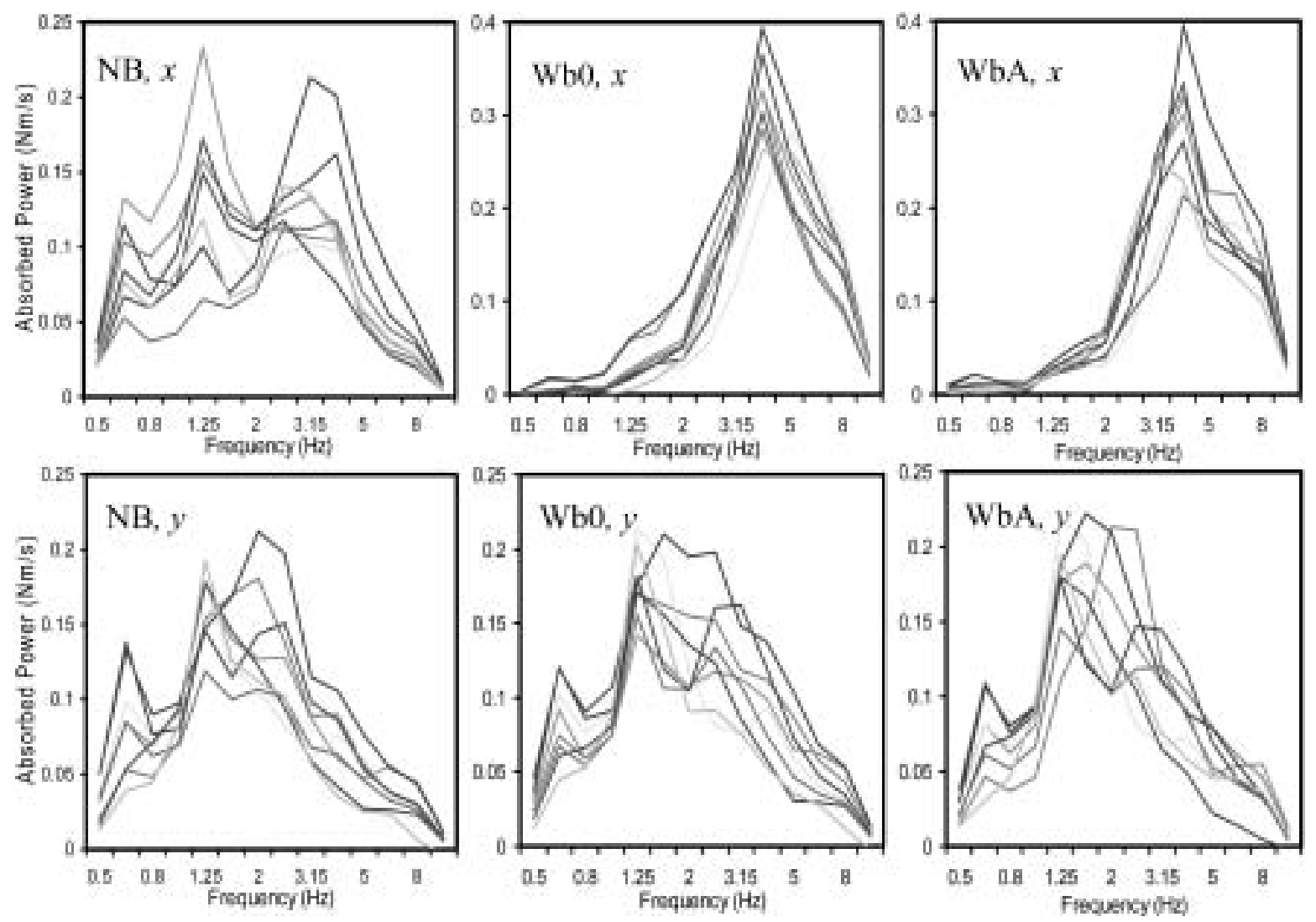

Fig. 3. Comparison of absorbed power magnitude responses measured at the seat pan of eight subjects seated with NB (no back support), Wb0 (vertical back support) and WbA: (inclined back support) postures, and exposed to $1 \mathrm{~m} / \mathrm{s}^{2} \mathrm{rms}$ acceleration along the fore-aft $(x)$ and lateral $(y)$ directions (seat height $\mathbf{H}_{1}$ ).

ably larger variability of the measured data is thus observed for the NB posture. The variability among the individual data set is greatly reduced with back supported postures, particularly under $x$-axis vibration. This may be attributed to more controlled sitting against the back support. The $P_{A b s}$ responses of seated occupants with the NB posture under $x$ - and $y$ - axis vibration reveal comparable frequencies corresponding to the peaks. The frequencies corresponding to peak magnitude responses under $x$ - and $y$ - axis vibration, however, differ considerably for the back supported postures.

The absorbed power responses clearly demonstrate important effects of the back support condition and direction of vibration, which are also evident from the reported APMS responses to $\mathrm{HV}^{14)}$. The effect of the back support condition appears to be far more important under $x$ - axis vibration. This effect, however, is quite small under $y$ - axis vibration, since the magnitudes of corresponding $y$-axis forces developed at the back support are relatively small. An inclined back support generally yields lower peak magnitudes under fore-aft vibration for the majority of participants compared to that obtained with the vertical back support.
The variability in the measured responses may be attributed to a number of factors, namely the body mass, body build and upper body adhesion with the back support $^{21,22)}$. The responses measured at the back support also show a similar degree of variability, as shown in Fig. 4, for the $\mathrm{WbO}$ and $\mathrm{WbA}$ postures. Owing to considerably lower dynamic interactions of the upper body with the backrest under $y$-axis vibration, the absorbed power responses tend to be substantially small compared to those obtained under $x$-axis vibration. Furthermore, the measured data reveals far greater variability under $y$-axis vibration. The low frequency $y$-axis vibration caused excessive side-to-side sliding of the upper body with respect to the back support. Subjects generally showed stiffening tendency to resist this motion. The greater variability in the measured data at lower frequencies was thus attributed to this body stiffening behaviour. The relatively lower magnitudes of absorbed power at some of the frequencies cause considerably higher values of the coefficient of variation $(\mathrm{CoV})$, even though the standard deviation of the mean could be small. Relatively higher values of $\mathrm{CoV}$, exceeding $100 \%$, were observed for measured responses along both directions in a few of the fre- 

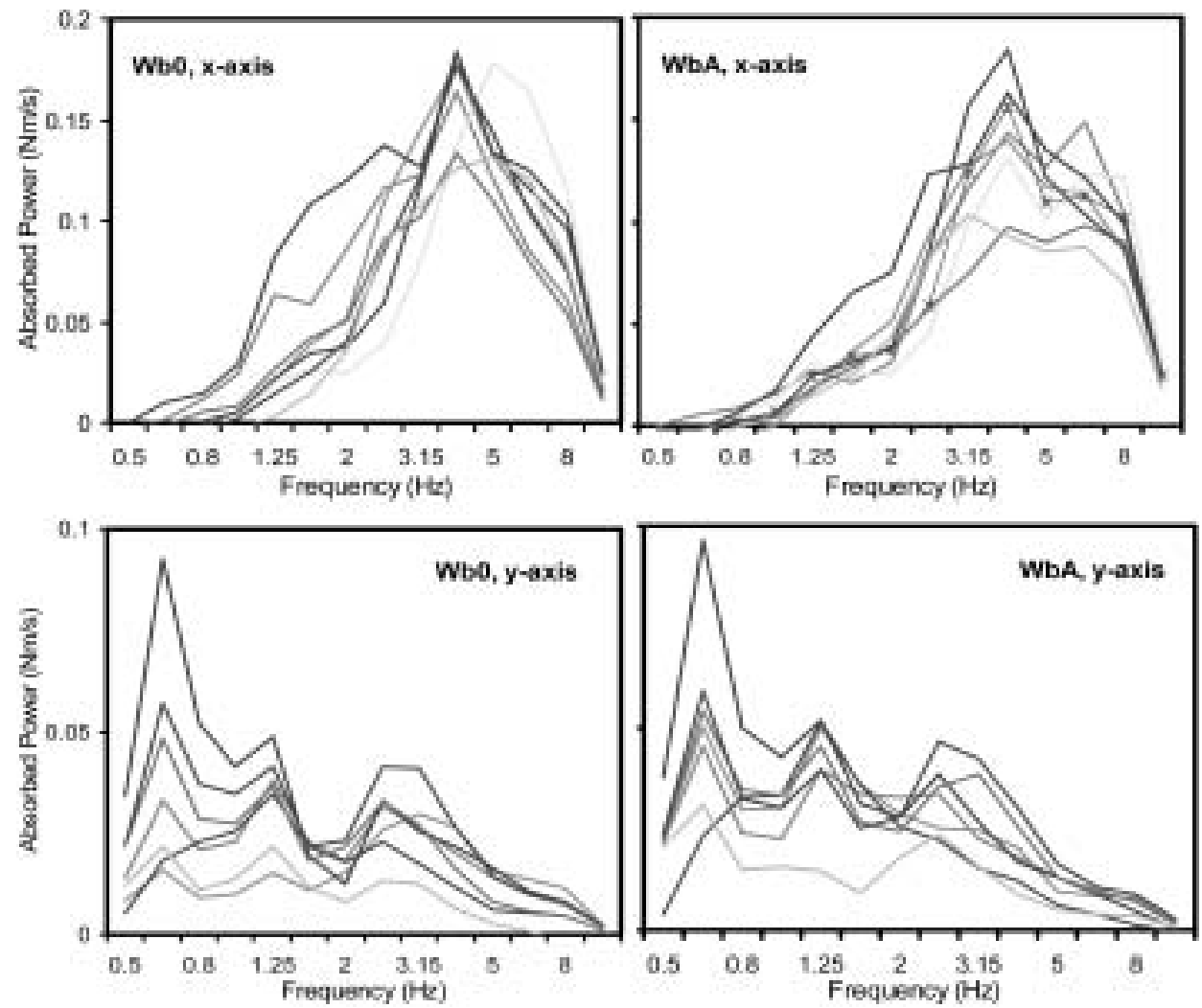

Fig. 4. Comparison of absorbed power magnitude responses measured at the backrest of eight subjects seated with Wb0 (vertical back support) and WbA (inclined back support) postures, and exposed to $1 \mathrm{~m} / \mathrm{s}^{2} \mathrm{rms}$ acceleration along the fore-aft $(x)$ and lateral $(y)$ directions (seat height $\mathbf{H}_{1}$ ).

quency bands, where the magnitudes were very small. Otherwise, the observed $\mathrm{CoV}$ values under $\mathrm{NB}$ sitting condition were found to be comparable with those reported for measured APMS responses under $\mathrm{HV}^{12,14)}$.

The results show that the peak magnitude responses of the subjects occur within narrow frequency ranges, and are strongly dependent upon sitting posture and direction of excitation. Thus the mean magnitudes in $1 / 3$-octave frequency bands are evaluated to study the important trends related to the effect of sitting posture, seat height and magnitude of vibration in view of both the seat pan and the backrest responses. Furthermore, the mean responses are considered to evaluate the effects of various factors.

Single-factor ANOVA, 'with-in subjects' were performed to identify the most significant factors affecting the absorbed power responses measured at the seat pan and the backrest. The analysis involved excitation magnitude, seat height and back support conditions as the main factors. Two-factor ANOVA were also performed to analyse the significance of interactions between the two contributing factors on the absorbed power response obtained at the seat pan and the backrest. Tables 3 and 4 summarize the results attained for the responses measured at the seat pan under $x$ - and $y$-axis excitations, respectively, corresponding to selected frequencies, considering the three levels each of the excitation magnitude and seat height, and their interactions, for each sitting posture. Owing to important observed effects of back support conditions, the significant differences in the measured absorbed power response at the seat pan are also evaluated for the two back-supported postures ( $\mathrm{Wb0} v s \mathrm{WbA}$ ) and all the three postures ( $\mathrm{NB} v s \mathrm{~Wb} 0 v s \mathrm{WbA}$ ). The results are summarized in Table 5 for both axes of vibration. Owing to relatively small magnitudes of measured power and greater $\mathrm{CoV}$ of the measured data, a few studies have employed either peak magnitudes or total absorbed power to study the effects of contributing factors $^{22,23)}$. In this study, the total absorbed power responses of 8 subjects were also evaluated from the one thirdoctave bands spectra, using Eq. (8). The CoV values of the total $P_{A b s}$ data were computed to analyze the intersubject variability. The $\mathrm{CoV}$ values up to $20 \%$ were obtained over the experimental conditions considered. Moreover, no particular trends in the values of $\mathrm{CoV}$ could be observed with respect to the magnitude of excitation, sitting posture, seat height or direction of excitation.

The absorbed power derived on the basis of dynamic 
Table 3. $p$-values attained from single and two-factor ANOVA performed on the seat pan absorbed power magnitude under fore-aft vibration

\begin{tabular}{c|lcc|ccc|lcc}
\hline Factor & \multicolumn{3}{|c|}{ Excitation $\left(.25, .5,1.0 \mathrm{~m} / \mathrm{s}^{2}\right)$} & \multicolumn{3}{c|}{ Height $\left(\mathrm{H}_{1}, \mathrm{H}_{2}, \mathrm{H}_{3}\right)$} & \multicolumn{3}{c}{ Excitation *Height } \\
\hline Frequency $(\mathrm{Hz})$ & $\mathrm{NB}^{\dagger}$ & $\mathrm{Wb0}$ & $\mathrm{WbA}^{\S}$ & $\mathrm{NB}$ & $\mathrm{Wb} 0$ & $\mathrm{WbA}$ & $\mathrm{NB}$ & $\mathrm{Wb0}$ & $\mathrm{WbA}$ \\
\hline 0.63 & 0.000 & 0.000 & 0.000 & 0.054 & 0.294 & 0.005 & 0.031 & 0.319 & 0.006 \\
0.75 & 0.000 & 0.000 & 0.000 & 0.223 & 0.508 & 0.017 & 0.005 & 0.365 & 0.011 \\
1 & 0.000 & 0.000 & 0.000 & 0.142 & 0.569 & 0.101 & 0.898 & 0.318 & 0.153 \\
1.13 & 0.000 & 0.000 & 0.000 & 0.386 & 0.557 & 0.335 & 0.532 & 0.756 & 0.546 \\
2 & 0.000 & 0.000 & 0.000 & 0.235 & 0.806 & 0.628 & 0.605 & 0.54 & 0.495 \\
3 & 0.000 & 0.000 & 0.000 & 0.597 & 0.176 & 0.392 & 0.929 & 0.371 & 0.372 \\
4 & 0.000 & 0.000 & 0.000 & 0.716 & 0.008 & 0.064 & 0.905 & 0.004 & 0.007 \\
5 & 0.000 & 0.000 & 0.000 & 0.401 & 0.054 & 0.070 & 0.792 & 0.076 & 0.053 \\
6 & 0.000 & 0.000 & 0.000 & 0.299 & 0.513 & 0.016 & 0.69 & 0.68 & 0.039 \\
8 & 0.000 & 0.000 & 0.000 & 0.899 & 0.504 & 0.007 & 0.915 & 0.782 & 0.000 \\
\hline
\end{tabular}

$\mathrm{NB}^{\dagger}$ - No back support; $\mathrm{WbO}^{\ddagger}$ - Vertical back support; $\mathrm{WbA}^{\S}$ - Inclined back support.

Table 4. $p$-values attained from single and two-factor ANOVA performed on the seat pan absorbed power magnitude under lateral vibration

\begin{tabular}{c|lcc|ccc|lcc}
\hline Factor & \multicolumn{2}{|l|}{ Excitation $\left(.25, .5,1.0 \mathrm{~m} / \mathrm{s}^{2}\right)$} & \multicolumn{3}{c|}{ Height $\left(\mathrm{H}_{1}, \mathrm{H}_{2}, \mathrm{H}_{3}\right)$} & \multicolumn{3}{c}{ Excitation *Height } \\
\hline Frequency $(\mathrm{Hz})$ & $\mathrm{NB}^{\dagger}$ & $\mathrm{Wb}^{\ddagger}$ & $\mathrm{WbA}^{\S}$ & $\mathrm{NB}$ & $\mathrm{Wb} 0$ & $\mathrm{WbA}$ & $\mathrm{NB}$ & $\mathrm{Wb0}$ & $\mathrm{WbA}$ \\
\hline 0.63 & 0.000 & 0.253 & 0.008 & 0.322 & 0.785 & 0.965 & 0.437 & 0.657 & 0.879 \\
0.75 & 0.000 & 0.000 & 0.000 & 0.382 & 0.178 & 0.747 & 0.811 & 0.25 & 0.568 \\
1 & 0.000 & 0.000 & 0.000 & 0.756 & 0.811 & 0.698 & 0.392 & 0.992 & 0.838 \\
1.13 & 0.000 & 0.000 & 0.000 & 0.081 & 0.927 & 0.861 & 0.051 & 0.962 & 0.976 \\
2 & 0.000 & 0.000 & 0.000 & 0.013 & 0.581 & 0.378 & 0.96 & 0.532 & 0.277 \\
3 & 0.000 & 0.000 & 0.000 & 0.381 & 0.215 & 0.302 & 0.616 & 0.035 & 0.216 \\
4 & 0.000 & 0.000 & 0.000 & 0.024 & 0.028 & 0.798 & 0.340 & 0.001 & 0.938 \\
5 & 0.000 & 0.000 & 0.000 & 0.001 & 0.226 & 0.669 & 0.009 & 0.153 & 0.931 \\
6 & 0.000 & 0.000 & 0.000 & 0.000 & 0.757 & 0.377 & 0.000 & 0.714 & 0.682 \\
8 & 0.000 & 0.000 & 0.000 & 0.000 & 0.036 & 0.313 & 0.000 & 0.088 & 0.746 \\
\hline
\end{tabular}

$\mathrm{NB}^{\dagger}$ - No back support; $\mathrm{Wb}^{\ddagger}-$ Vertical back support; $\mathrm{WbA}^{\S}$ - Inclined back support.

Table 5. Effect of posture shown by the $p$-values derived from single-factor ANOVA performed on the seat pan absorbed power magnitude data under Fore-and-aft and lateral excitations

\begin{tabular}{c|c|ccccccccccc}
\hline Axis & Frequency $(\mathrm{Hz})$ & 0.63 & 0.75 & 1 & 1.13 & 2 & 2.75 & 4 & 5 & 6 & 8 \\
\hline \multirow{2}{*}{$x-$} & Wb0vsWbA & 0.003 & 0.121 & 0.388 & 0.830 & 0.054 & 0.495 & 0.000 & 0.000 & 0.027 & 0.038 \\
& NBvsWb0vsWbA & 0.000 & 0.000 & 0.702 & 0.036 & 0.157 & 0.004 & 0.000 & 0.000 & 0.000 & 0.000 \\
\hline \multirow{2}{*}{$y-$} & Wb0vsWbA & 0.064 & 0.001 & 0.278 & 0.094 & 0.004 & 0.009 & 0.906 & 0.000 & 0.000 & 0.250 \\
& NBvsWb0vsWbA & 0.000 & 0.000 & 0.000 & 0.000 & 0.000 & 0.125 & 0.000 & 0.000 & 0.000 & 0.000 \\
\hline
\end{tabular}

NB - No back support; Wb0 - Vertical back support; WbA — Inclined back support.

interactions at the seat pan driving-point can be considered to represent the total energy transferred to the body, while that resulting from backrest driving-point may be interpreted as energy transferred to the upper body from the seat pan and the back support. In order to quantify the effect of the back support, the ratio of the total power absorbed at the backrest to that at the seat pan is computed, such that:

$$
P_{\gamma x}=\frac{\overline{P_{b x}}}{\overline{P_{p x}}} \text { and } P_{\gamma y}=\frac{\overline{P_{b y}}}{\overline{P_{p y}}}
$$

Where $P_{\gamma k}(k=x, y)$ is the ratio of total power derived from the seat back response to that from the seat pan response under excitations along axis $k$. The total power measured at the seat pan and the backrest, together with absorbed power ratio $\left(\mathrm{APR}, P_{\gamma k}\right.$ ) are summarized in Table 6 for 
Table 6. The total absorbed power measured at the seat pan and the backrest, under the influence of various unsupported and supported back postures, and magnitudes of vibration at seat height $\mathbf{4 2 5} \mathbf{~ m m}$

\begin{tabular}{|c|c|c|c|c|c|c|c|c|}
\hline \multirow{3}{*}{ Axis } & \multirow{3}{*}{$\begin{array}{c}\text { Vibration } \\
\text { Level }\end{array}$} & \multicolumn{5}{|c|}{ Total absorbed power $(\mathrm{Nm} / \mathrm{s})$} & \multirow{2}{*}{\multicolumn{2}{|c|}{ APR $P \gamma$}} \\
\hline & & \multicolumn{3}{|c|}{ Seat Pan } & \multicolumn{2}{|c|}{ Backrest } & & \\
\hline & & $\mathrm{NB}^{\dagger}$ & $\mathrm{Wb} 0^{\ddagger}$ & $\mathrm{WbA}^{\S}$ & $\mathrm{Wb} 0$ & $\mathrm{WbA}$ & $\mathrm{Wb} 0$ & WbAJ \\
\hline \multirow{3}{*}{$x$} & $0.25 \mathrm{~m} / \mathrm{s}^{2}$ & 0.09 & 0.08 & 0.07 & 0.05 & 0.04 & 59.93 & 58.08 \\
\hline & $0.5 \mathrm{~m} / \mathrm{s}^{2}$ & 0.32 & 0.34 & 0.32 & 0.21 & 0.19 & 60.64 & 57.95 \\
\hline & $1.0 \mathrm{~m} / \mathrm{s}^{2}$ & 1.14 & 1.38 & 1.32 & 0.85 & 0.78 & 61.83 & 59.27 \\
\hline \multirow{3}{*}{$y$} & $0.25 \mathrm{~m} / \mathrm{s}^{2}$ & 0.09 & 0.09 & 0.09 & 0.04 & 0.04 & 38.49 & 40.56 \\
\hline & $0.5 \mathrm{~m} / \mathrm{s}^{2}$ & 0.31 & 0.34 & 0.34 & 0.11 & 0.12 & 31.31 & 36.04 \\
\hline & $1.0 \mathrm{~m} / \mathrm{s}^{2}$ & 1.09 & 1.20 & 1.18 & 0.31 & 0.36 & 25.85 & 30.14 \\
\hline
\end{tabular}

$\mathrm{NB}^{\dagger}$ - No back support; $\mathrm{WbO}^{\ddagger}$ - Vertical back support; $\mathrm{WbA}^{\S}$ - Inclined back support.

both axes of vibration, under the influence of unsupported and supported back postures and magnitudes of vibration. Considering the relatively small effect of seat height in most of the frequency range (Tables 3 and 4), the results are presented for the seat height of $425 \mathrm{~mm}\left(\mathrm{H}_{1}\right)$. Further analyses of the absorbed power spectra revealed that the energy transfer in frequency bands corresponding to the principal resonances account for nearly $70 \%$ and $90 \%$ of the total absorbed power in the $0.5-10 \mathrm{~Hz}$ frequency range, under the $x$ - and $y$ - axis vibration, respectively. This suggests relatively larger deformations of musculoskeletal structure around the principle resonances, and thus the greater energy dissipation.

\section{Normalization of the measured absorbed power response}

The magnitudes of the total absorbed power obtained at the seat pan $\left(\overline{P_{p x}}\right)$ and backrest $\left(\overline{P_{b x}}\right)$ revealed superior correlation $\left(r^{2}>0.8\right)$ with the body mass for both the back-supported postures and all three levels of fore-aft vibration. Similar degree of correlation of $\overline{P_{p y}}$ with the body mass was also observed under $y$-axis vibration $\left(r^{2}\right.$ $>0.9$ ), while extremely poor correlation was obtained for $\overline{P_{b y}}\left(r^{2}<0.2\right)$. This could be attributed to relatively small magnitudes of lateral forces developed at the upper bodyseat back interface and sliding of the upper body against the backrest under $y$-axis vibration.

The normalizations of absorbed power spectra with respect to the seated mass resulted in nearly $10 \%$ reduction in $\mathrm{CoV}$ values at frequencies above $2.5 \mathrm{~Hz}$ for the NB posture, while only negligible effects were observed in the $1-2.5 \mathrm{~Hz}$ frequency range. The effect of normalization on the $\mathrm{CoV}$ of the absorbed power spectra with back supported postures was noticed only near frequencies corresponding to the peaks. Consequently, the subsequent analyses of the measured data were performed without the normalization, which allows for interpretations of the contributing factors on the basis of the unbiased frequency responses.

\section{Effect of vibration magnitude}

The reported studies ${ }^{20,22-24)}$ both under vertical and HV have consistently concluded that the total absorbed power increases approximately in proportion to the square of the excitation magnitude. The strong effects of the vibration magnitude are most likely attributed to many factors, such as, the nonlinear behaviour of the seated body, excessive upper body movements under higher excitations, increase in shifting tendencies of the occupants to realize more stable posture under higher vibration magnitudes, and contributions due to the legs. The across subjects mean responses attained with $\mathrm{NB}$ and WbA postures $(n=8)$, under different magnitudes $x$ - and $y$-axis excitations, obtained at the seat pan and the backrest, respectively, are illustrated in Figs. 5 and 6. The figures show mean responses to 0.5 and $1.0 \mathrm{~m} / \mathrm{s}^{2}$ excitations, while those to $0.25 \mathrm{~m} / \mathrm{s}^{2} \mathrm{rms}$ acceleration excitation are omitted due to very small power values. Furthermore, the mean responses attained with $\mathrm{Wb} 0$ posture are also omitted, since these were quite similar to those obtained with WbA posture. The results clearly show strong and nonlinear effects of vibration magnitude on both the seat pan and backrest responses, irrespective of the direction of excitation. The strong influence of the vibration magnitude is also evident from the results attained from ANOVA, presented in Tables 3 and 4 for the $x$ - and $y$-axes, respectively, where $p<0.005$ in most of the frequency range. The effect of vibration magnitude on the power derived from seat pan interactions is highly significant $(p<0.001)$ in the entire frequency range under both axes of vibration and all the three sitting postures.

The total absorbed power responses derived from the two driving-points under both axes of vibration suggest a nearly quadratic relation with the magnitude of vibration, which may be expressed in the form:

$\bar{P}=\alpha a^{\beta}$ 

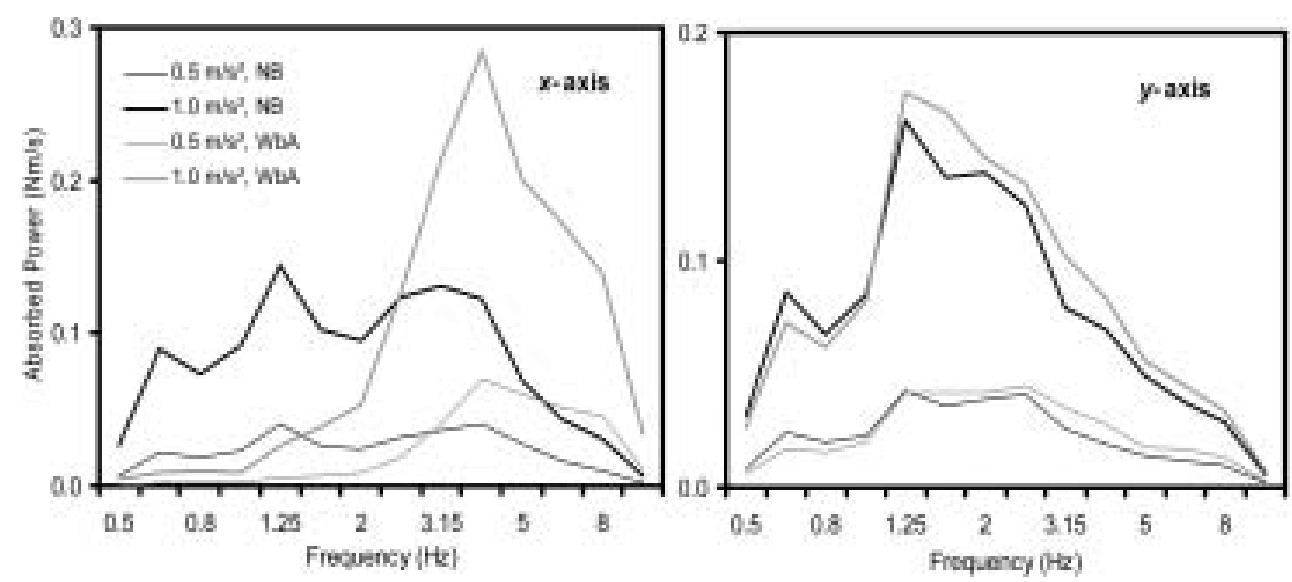

Fig. 5. Mean across subjects $(n=8)$ absorbed power characteristics measured at the seat pan under different magnitudes of excitation 0.5 and $1.0 \mathrm{~m} / \mathrm{s}^{2} \mathrm{rms}$, and, NB (no back support) and WbA (inclined back support) postures, along fore-aft $(x)$ and lateral $(y)$ vibration.

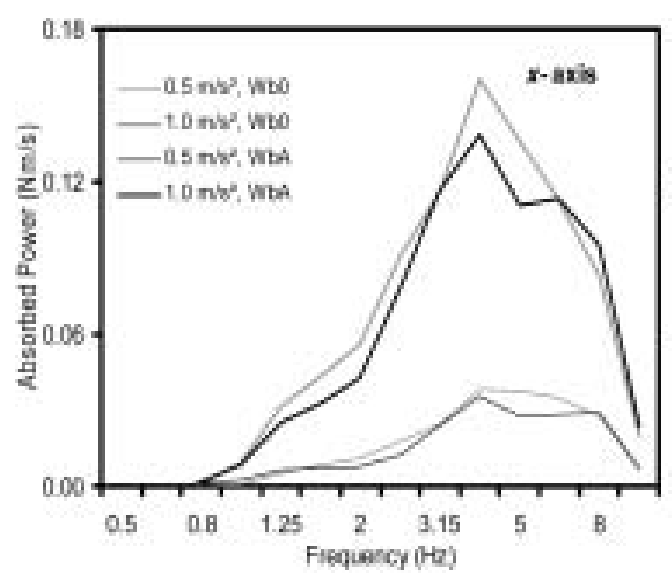

(a)

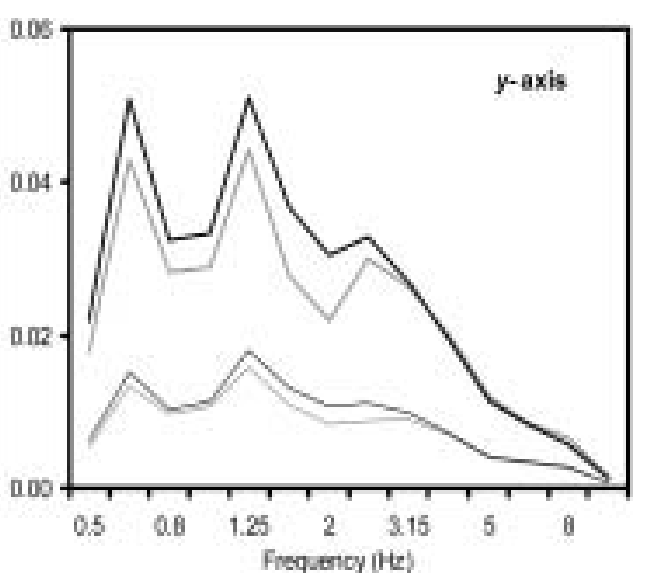

(b)

Fig. 6. Mean across subjects $(n=8)$ absorbed power characteristics measured at the backrest under different magnitudes of excitation 0.5 and $1.0 \mathrm{~m} / \mathrm{s}^{2} \mathrm{rms}$, and Wb0: (vertical back support) and WbA (inclined back support) postures: a) fore-aft $(x)$ vibration; b) lateral $(y)$ vibration.

Table 7. Constant and exponent values for different excitation and postural conditions

\begin{tabular}{cccccccccc}
\hline \multirow{2}{*}{ Location } & \multirow{2}{*}{ Axis of vibration } & \multicolumn{3}{c}{ exponent $\beta$} & & \multicolumn{3}{c}{ constant $\alpha$} \\
\cline { 9 - 10 } \cline { 7 - 9 } & & $\mathrm{NB}^{\dagger}$ & $\mathrm{Wb}^{\ddagger}$ & $\mathrm{WbA}^{\S}$ & & $\mathrm{NB}$ & $\mathrm{Wb} 0$ & $\mathrm{WbA}$ \\
\hline \multirow{2}{*}{ Seat pan } & $\mathrm{x}$ & 1.84 & 2.05 & 2.07 & & 1.15 & 1.39 & 1.33 \\
& $\mathrm{y}$ & 1.81 & 1.85 & 1.86 & & 1.09 & 1.21 & 1.19 \\
\hline \multirow{2}{*}{ Backrest } & $\mathrm{x}$ & - & 2.07 & 2.08 & & - & 0.86 & 0.79 \\
& $\mathrm{y}$ & - & 1.56 & 1.64 & & - & 0.31 & 0.36 \\
\hline
\end{tabular}

$\mathrm{NB}^{\dagger}$ - No back support; $\mathrm{Wb}^{\ddagger}$ - Vertical back support; $\mathrm{WbA}^{\S}$ - Inclined back support

Where $\beta$ is the exponent of the overall rms acceleration $a$ due to excitation and $\alpha$ is the proportionality constant. The regression analysis of the measured total power data resulted in a correlation coefficient $\left(r^{2}\right)$ in excess of 0.9 for all the experimental conditions considered. Table 7 summarizes the constant and the exponent values obtained for different excitation and postural conditions. The expo- nent values for the pan measured power range from 1.84-2.07 and 1.81-1.86 under $x$ - and $y$-axis vibration, respectively. The corresponding values of the constants range from 1.15-1.39 and 1.09-1.21, respectively. Both the constant and exponent values tend to be higher for the back supported postures compared to those for the NB posture under fore-aft vibration, suggesting greater depen- 
dence of the total energy transfer on the vibration magnitude when the upper body is supported against a backrest. Under $y$-axis vibration, the exponent value for the NB posture tends to be only slightly smaller compared to those for the $\mathrm{WbO}$ and $\mathrm{WbA}$ postures, while the difference in the proportionality constant is considerable. These suggest that energy transfer to the body increases at a greater rate of excitation acceleration, when the upper body is supported under both axes of HV. This may be partly attributed to the fact that contact with the back serves as an additional driving-point or source of vibration.

The results further show relatively lower values of the exponent and the constant under lateral axis excitation, compared to the fore-aft vibration. These suggest that relatively smaller amount of energy is dissipated within the body exposed to lateral vibration, compared to that under identical magnitude of fore-aft vibration, which encourages greater interactions of the upper body with the back support. This is also evident from the total power derived from back support interactions, which tends to considerably higher under $x$-axis vibration. The exponent values for the $\overline{P_{b x}}$ range from 2.07 to 2.08 under $x$-axis vibration, which are considerably larger than those for the $\overline{P_{b y}}(1.56-1.64)$ under $y$-axis vibration. Even larger difference is evident from the constant values, which range from 0.79 to 0.86 and 0.31 to 0.36 under $x$ - and $y$-axes vibration, respectively, which further attest to enhanced interactions of the upper body with the back support under fore-aft motions. This is further evident from the absorbed power ratio (APR), which lies in the 58-62\% range for the fore-aft vibration but is only $25-40 \%$ under lateral vibration of different magnitudes considered in the study. Reduced power measured at the back support under lateral vibration can be attributed to relatively smaller magnitude of force developed along the $y$-axis, and lower resistance provided by the backrest to limit the upper body motion. It is essential to note that variations in seat height revealed only minimal effect of the total power, irrespective of the excitation magnitude and back support condition.

\section{Effect of posture}

The results show most important effect of back support on the absorbed power responses measured at the seat base under $x$ - axis vibration, while the effect under $y$-axis is very small, as seen in Fig. 5 and Table 5. The results show significant effects of posture on the seat pan-measured power $(p<0.001)$, when the variations are considered for all three postures, except in a few frequency bands in the $x$-axis response. The variations in the back support ( $\mathrm{Wb} 0$ vs $\mathrm{WbA}$ ) also show effect on the $x$-axis response at frequencies above $2.75 \mathrm{~Hz}(p<0.05)$, while the effect is more evident above $4 \mathrm{~Hz}$ under the $y$-axis motion. Owing to the very low power magnitudes under $0.25 \mathrm{~m} / \mathrm{s}^{2}$ excitation and relatively smaller effects of the two back supported postures, the figure illustrates comparisons of the results attained with $\mathrm{NB}$ and $\mathrm{WbA}$ conditions, and two magnitudes of vibration. Figure 6 further shows the comparisons of spectra of absorbed power for the two back support conditions, derived from the back support driving-point.

The influences of the back support conditions, considered in this study, on the mean absorbed power spectra in the $0.5-10 \mathrm{~Hz}$ frequency range under $x$ - and $y$-axis vibration of magnitude of $1.0 \mathrm{~m} / \mathrm{s}^{2}$ are further illustrated in Fig. 7. For the NB condition, the absorbed power response under $x$ - axis vibration reveals peaks in the fre-

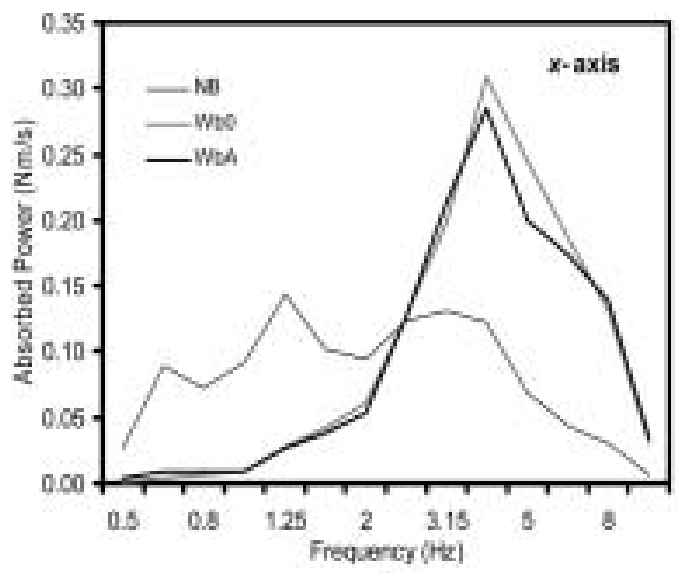

(a)

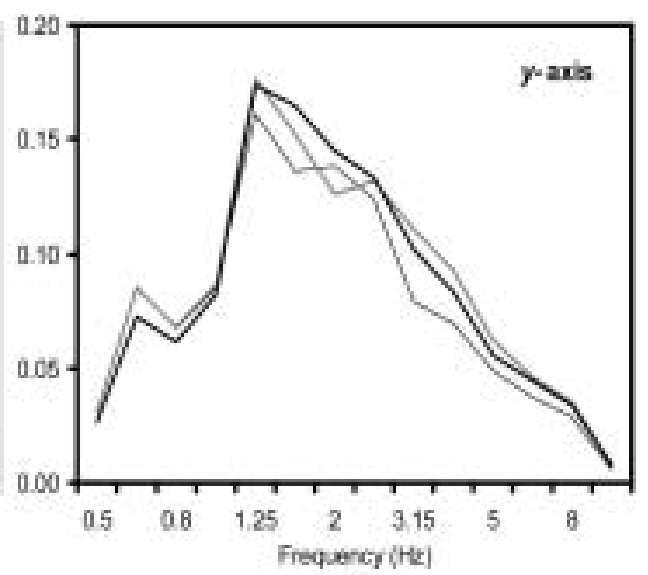

(b)

Fig. 7. Mean absorbed power characteristics $(n=8)$ measured at the seat pan under NB (no back support) Wb0 (vertical back support) and WbA (inclined back support) postures (excitation magnitude $1.0 \mathrm{~m} / \mathrm{s}^{2} \mathrm{rms}$ ): a) fore-aft (x) vibration; b) lateral (y) vibration. 
quency bands centered around $0.63,1.25$ and $3.15 \mathrm{~Hz}$, while the response under $y$-axis vibration reveal peaks near $0.63,1.25$ and $2 \mathrm{~Hz}$. These frequencies corresponding to the peaks are comparable to those observed in the APMS responses of seated occupants exposed to $\mathrm{HV}^{14)}$. It has been reported that the primary resonance occurring at lower frequencies is due to pitch motion of the upper body ${ }^{15}$. An analysis of total power absorbed further showed that nearly $50 \%$ of the total power under an NB posture is absorbed in the lower frequency range of $0.5-2.19 \mathrm{~Hz}$, and can be attributed to the upper body motion. The upper body restrained against a vertical or inclined backrest reveals peak magnitude at a considerably higher frequency under the fore-aft motion, as seen in Fig. 7. The dominant peak response shifts to center frequency of $4 \mathrm{~Hz}$, when the back is supported, while the peak magnitude tends to be considerably larger. A slight secondary peak is also observed in the band centered around $6.3 \mathrm{~Hz}$ for the $\mathrm{WbA}$ posture. This suggests that the use of a back support helps to stiffen the upper body under fore-aft motion. This tendency, however, is not evident under lateral vibration. The total power attained from the pan measurements suggest comparable total power of the 3 sitting conditions under both axes of vibration of smaller magnitudes $\left(0.25\right.$ and $\left.0.5 \mathrm{~m} / \mathrm{s}^{2}\right)$. An increase in the excitation magnitude, however, seems to cause greater energy transfer for the back supported postures. Under the $1 \mathrm{~m} / \mathrm{s}^{2}$ fore-aft excitation, nearly 16-20\% larger energy is transferred to the body with the back support than the NB sitting condition (Table 6). This increase reduces to approximately $10 \%$ for the lateral acceleration excitation of the same magnitude.

Figure 8 illustrates a comparison of the 1/3-octave band spectra of powers measured at the seat pan and the back support (WbA) under fore-aft motion. The two responses show peaks in the identical frequency bands centered around 4 and $6.3 \mathrm{~Hz}$. An inclined back support would

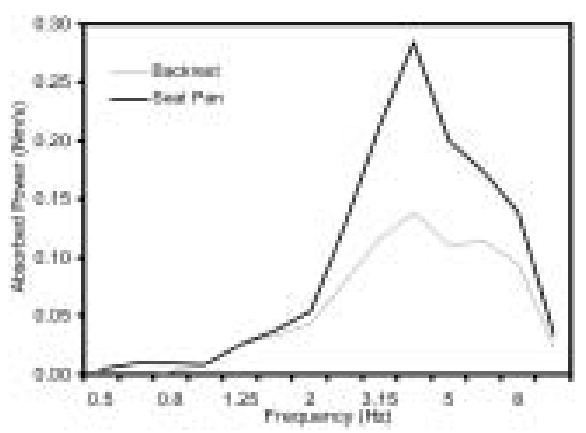

Fig. 8. Comparison of mean absorbed power response $(n=8)$ measured at the seat pan and the backrest under fore-and-aft $(x)$ vibration (WbA - inclined back supported posture, Seat height-H1). impose relatively less vibration to the upper body along a direction normal to the contact surface, and could thus yield slightly lower absorbed power. The response measured at the backrest along the fore-aft direction show that a $\mathrm{WbA}$ posture yields slightly lower values of total power measured at the back support and APR than the $\mathrm{Wb} 0$ posture (Table 6). This may be attributed to more stable upper body support in the WbA condition. An inclined back support tends to limit the backward upper body motion, while the forward motion is limited by the weight of the subject resting against the back support. The measured power responses under the lateral motion, however, show an opposite trends, where the APR values for the $\mathrm{WbA}$ posture tend to be slightly higher than the $\mathrm{Wb} 0$ posture, irrespective of the excitation magnitude. This may be attributed to relatively less sliding of the upper body against the inclined back support.

\section{Effect of seat height}

The absorbed responses revealed very small effect of the seat height, irrespective of the back support condition and excitation magnitude. The effect was particularly negligible for the NB and Wb0 sitting conditions $(p>0.05)$ when exposed to fore-aft vibration, and for $\mathrm{WbA}$ posture under lateral vibration. The NB posture showed only small influence of the seat height under lateral vibration at frequencies above $4 \mathrm{~Hz}(p<0.05)$, as seen in Table 4 .

\section{Discussions in view of the reported results}

The absorbed power characteristics of the seated human exposed to HV have been reported in a single study ${ }^{23)}$. This study considered only NB posture with feet resting on a stationary support and thus not vibrated. The study considered different magnitudes of sinusoidal fore-aft and lateral vibration $\left(0.25-1.4 \mathrm{~m} / \mathrm{s}^{2} \mathrm{rms}\right)$ at discrete frequencies in the $1.13-20 \mathrm{~Hz}$ range with steps of $1 / 6$ octaves and reported that peak responses occur below $3 \mathrm{~Hz}$. The present study considered sitting with back supported and unsupported conditions on a rigid seat with three different heights, while exposed to three levels of white noise random vibration in the $0.5-10 \mathrm{~Hz}$ range. The mean responses of the seated body with $\mathrm{NB}$ posture revealed primary resonant peaks near 0.63 and $1.25 \mathrm{~Hz}$ under each axis of vibration. An additional peak in the $3.15 \mathrm{~Hz}$ band was also observed under fore-aft vibration. The lower frequency peaks could not be observed in the reported study, since it considered vibration at $1.13 \mathrm{~Hz}$ and above, while a peak in the fore-aft response near $2.5 \mathrm{~Hz}$ was evident. Moreover, the reported study was conducted under sinusoidal vibration, which could yield different magnitudes of absorbed power than those observed under random vibration. The differences in the responses are also partly attributable to the stationary legs support used in 
the reported study. Under exposure to HV, the occupants' legs are expected to undergo relative movements and thus contribute to energy transfer. Similar to another reported study under vertical vibration ${ }^{22}$, the total power obtained in this study at both the seat pan and backrest revealed good correlation with the body mass under the experimental conditions considered, irrespective of the axis of vibration. The mean total power measured under $1.0 \mathrm{~m} / \mathrm{s}^{2} \mathrm{rms}$ vertical vibration $(0.5-15 \mathrm{~Hz})$ with $\mathrm{NB}$ posture and hands in lap was reported as $0.2 \mathrm{~W}^{22)}$, the present study revealed total power under the same posture and $\mathrm{HV}$ magnitude $(0.5-10 \mathrm{~Hz})$ in the order of $1.1 \mathrm{~W}$. Considering the differences in the frequency bands used in the two studies and thus the magnitude, these results suggest that the energy transfer to the body under HV could be equally important when compared to that under vertical vibration.

\section{A discussion on frequency-weighting of vibration power} absorption (VPA)

According to ISO 2631-18), the frequency-weighted acceleration $\left(a_{w}\right)$ at a frequency $(\omega)$ for a given vibration acceleration $(a)$ is calculated from

$a_{w}(\omega)=W_{d}(\omega) \cdot a(\omega)$

where $W_{d}$ is the frequency weighting function defined in ISO 2631-18).

In order to make a direct comparison of the VPA and the ISO-weighted acceleration, the VPA must be transferred to a function with the same form as that shown in Eq. (11) ${ }^{31)}$. As it is evident in Eq. (10), the absorbed power is statistically proportional to $a^{\beta}$, Therefore, the required proportional function is obtained by taking $\beta$ root of the $P_{A b s}$. The resulting function is further normalized to a reference value using the methodology proposed by Dong et al. ${ }^{31)}$, so that the VPA-based vibration measure has the same form as that shown in Eq. (11) and the VPA-based frequency weighting is directly comparable with the ISO $W_{d}$-weighting. The resulting VPA-based weighting is expressed as follows:

$$
W_{p j}(\omega)=\sqrt[\beta]{\frac{P_{p j}(\omega) / a_{p j}^{\beta}(\omega)}{\left(P_{p j} / a_{p j}^{\beta}\right)_{\operatorname{Re}_{f}}}} ; j=x, y
$$

where $W_{p j}$ is the magnitude of the weighting filter derived from spectra of power measured at the pan under vibration along direction $j(j=x, y), a_{p j}$ is the root-mean-square value of the acceleration input to the body in the VPA measurement, and $\sqrt[\beta]{\left(P_{p j} / a_{p j}^{\beta}\right)_{\operatorname{Re} f}}$ is the reference value of the non-normalized VPA weighting, which can be selected based on the purpose of the weighting application.

With the VPA-based frequency weighting, the VPA- based vibration measure $\left(a_{V P A}\right)$ is expressed as follows ${ }^{31)}$ :

$a_{V P A}(\omega)=W_{p j}(\omega) \cdot a(\omega)$

This equation clearly demonstrates that the VPA-based vibration measure is indeed composed of two components: (i) the vibration hazard represented by the input acceleration; and (ii) the biodynamic response represented by the VPA-based frequency weighting. An earlier study by Dong et al. ${ }^{31)}$ has shown that the VPA-based frequency weighting may also be derived from the driving-point mechanical impedance or apparent mass.

Since the VPA-based weighted acceleration in Eq. (13) and the ISO-weighted acceleration in Eq. (11) have the same form and the same vibration hazard or acceleration, the comparison of the two weightings can be used to identify the difference or similarity between the two measures. For the purpose of comparison, the VPA-based frequency weighting was computed using the $\beta$ values defined in Table 7 and the mean power spectra corresponding to 0.5 and $1.0 \mathrm{~m} / \mathrm{s}^{2}$ excitations along each axis. Each of the VPA-based frequency weightings is normalized to its peak value, while the maximum weighting is taken as unity. Figure 9 illustrates comparisons of magnitudes of
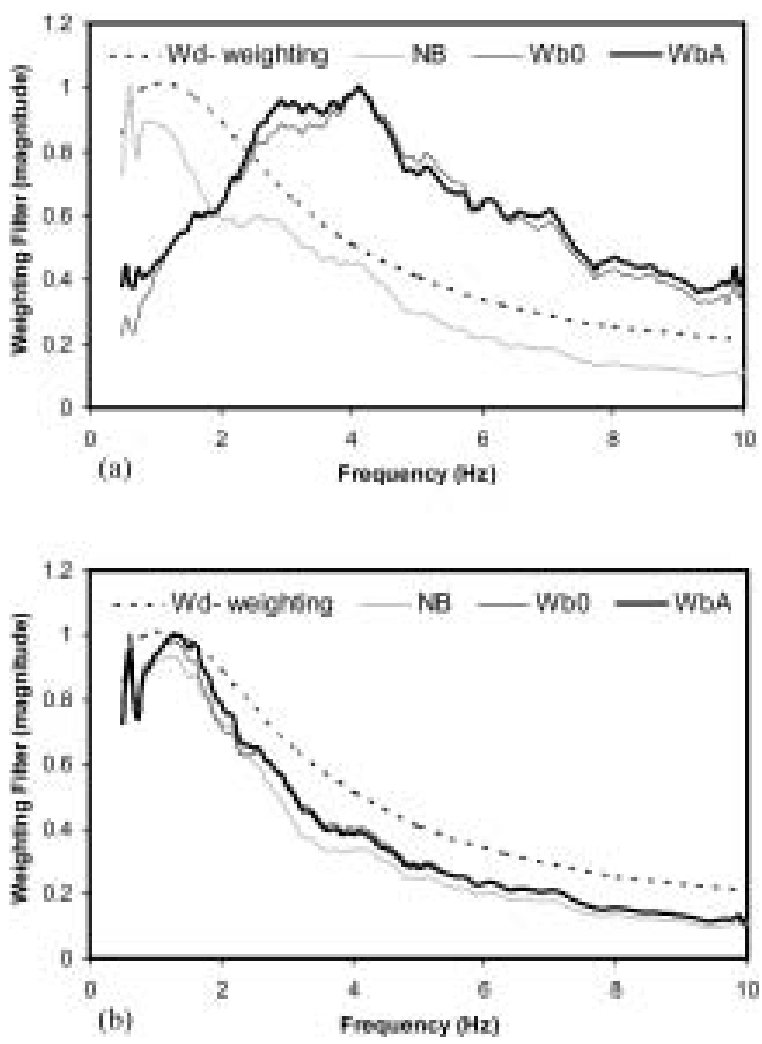

Fig. 9. Comparisons of weighting filter magnitudes derived from mean absorbed power responses corresponding to NB (no back support), Wb0 (vertical back support) and WbA: (inclined back support) postures (a) Fore-aft ( $x$ ) vibration; and (b) Lateral vibration. 
Table 8. Comparisons of the weighting values obtained in this study with the $\mathrm{W}_{d}$-weighting defined in ISO-2631-1 ${ }^{8}$

\begin{tabular}{c|c|ccc|ccc}
\hline \multirow{2}{*}{ Frequency (Hz) } & \multirow{2}{*}{$\mathrm{W}_{\mathrm{d}^{-} \text {-weighting }}$} & \multicolumn{3}{|c|}{ Fore-aft } & \multicolumn{3}{c}{ Lateral } \\
\cline { 3 - 8 } & & $\mathrm{NB}^{\dagger}$ & $\mathrm{Wb} 0^{\ddagger}$ & $\mathrm{WbA}^{\S}$ & $\mathrm{NB}^{\dagger}$ & $\mathrm{Wb}^{\ddagger}$ & WbA $^{\S}$ \\
\hline 0.5 & 0.853 & 0.746 & 0.214 & 0.373 & 0.651 & 0.635 & 0.556 \\
.63 & 0.944 & 1 & 0.243 & 0.375 & 1 & 1 & 0.901 \\
0.8 & 0.992 & 0.89 & 0.319 & 0.4 & 0.806 & 0.814 & 0.769 \\
1 & 1.011 & 0.899 & 0.4 & 0.438 & 0.854 & 0.895 & 0.880 \\
1.25 & 1.008 & 0.871 & 0.498 & 0.500 & 0.886 & 0.962 & 0.994 \\
1.6 & 0.968 & 0.741 & 0.604 & 0.596 & 0.775 & 0.836 & 0.932 \\
2 & 0.89 & 0.619 & 0.632 & 0.63 & 0.573 & 0.544 & 0.638 \\
2.5 & 0.776 & 0.614 & 0.788 & 0.871 & 0.402 & 0.428 & 0.450 \\
3.15 & 0.642 & 0.606 & 0.885 & 0.941 & 0.187 & 0.267 & 0.253 \\
4 & 0.512 & 0.482 & 1 & 1 & 0.143 & 0.188 & 0.174 \\
5 & 0.409 & 0.32 & 0.761 & 0.723 & 0.078 & 0.1 & 0.093 \\
6.3 & 0.323 & 0.225 & 0.588 & 0.596 & 0.047 & 0.058 & 0.057 \\
8 & 0.253 & 0.161 & 0.42 & 0.459 & 0.026 & 0.033 & 0.032 \\
10 & 0.212 & 0.138 & 0.366 & 0.374 & 0.019 & 0.024 & 0.017 \\
\hline
\end{tabular}

$\mathrm{NB}^{\dagger}$ - No back support; $\mathrm{Wb}^{\ddagger}-$ Vertical back support; $\mathrm{WbA}^{\S}$ - Inclined back support.

$W_{p j}$ attained for the three sitting conditions and $1 \mathrm{~m} / \mathrm{s}^{2}$ excitation along each direction of vibration with the current $W_{d}$-weighting defined in ISO-2631-18). The magnitudes of $W_{p j}$ and $W_{d}$-weightings are further compared and summarized in Table 8 in the $0.5-10 \mathrm{~Hz}$ frequency range.

As shown in Fig. 9, the two vibration magnitudes resulted in very similar values of the VPA-based frequency weighting function. This suggests that the VPAbased frequency weighting is not very sensitive to the variation of the vibration magnitude. The results also show that the general trend of the weighting function derived from the absorbed power responses to side-to-side vibration, $W_{p y}$, is generally consistent with that of $W_{d^{-}}$ weighting function, as seen in Fig. 9(b). The $W_{d}$-weighting also corresponds reasonably well with the $W_{p x}$ derived from the fore-aft VPA corresponding to the sitting condition without using the back support, as seen in Fig. 9(a). Since $W_{d}$ is derived primarily based on the subjective comfort data ${ }^{32)}$ measured for a sitting posture comparable to the NB posture used in the VPA measurement, the reasonably good agreement between the VPA-based and the ISO weightings suggests that the vibration power absorption in the horizontal vibration could be associated with the vibration sensation or perception for the NB posture. This reveals that the VPA could serve as an important measure of the vibration perception, which has also been observed in the hand-transmitted vibration exposure along the forearm direction ${ }^{31}$. Considering that the ISOweighted acceleration is an acceptable measure for assessing a health effect, the VPA may also be associated with the same health effect. These observations support the general hypothesis presented in the introduction section of this paper.

In the current ISO standard, the same $W_{d}$-weighting function is recommended for assessing the discomfort and health effects in the horizontal vibration exposure without differentiating the sitting postures with or without the back support. The results of this study suggest that this may not be a good practice. As shown in Fig. 9(a), the VPA-based fore-aft weightings $\left(W_{p x}\right)$ with back support differ greatly from that without the back support, as well as the $W_{d}$-weighting. This is attributed to greater interactions of the upper body with the back support under fore-aft motion. The biodynamic responses under these two sitting postures have been shown to be significantly different ${ }^{14,15)}$. Consequently, the resulting vibration perception or health effect should be different for the two postures. The frequency weightings used for assessing the vibration exposure for different back support conditions should also differ. Considering that the VPA measured under the sitting posture with the back support is likely to have some association with the discomfort or a health effect under such a sitting posture, the VPA-based frequency weighting for this posture may be used as one of the references for the development of an improved weighting for the risk assessment of the exposure under this posture. However, further studies of the comfort and health effects under this posture are required to test this VPA-based frequency weighting.

\section{Conclusions}

The absorbed power response characteristics of seated human subjects exposed to fore-aft and lateral vibration 
reveal considerable dynamic interactions between the upper body and the seat backrest, apart from those of the body and the seat pan. The results show that the vibration energy transferred and dissipated within the exposed body is strongly influenced by the back support condition, excitation magnitude and individual body mass, while the effect of seat height is nearly negligible. The responses measured at the seat pan and the backrest clearly show most important influences of the back support, particularly under fore-aft vibration. The absorbed power responses of the body seated without a back support dominate in the low frequency bands of 0.63 and $1.25 \mathrm{~Hz}$ under both axes of vibration, associated with the rocking and swaying motions of the upper body. An additional peak is also observed in the vicinity of 3.15 and $2 \mathrm{~Hz}$ under $x$ - and $y$-axis motion, respectively. These frequencies are similar to those observed from the reported apparent mass responses to HV. The presence of a backrest helps to stabilize the sitting posture by limiting the motions of the upper body, particularly under fore-aft excitations, and thereby stiffening of the body. The dominant responses of the body seated with a back support and exposed to fore-aft vibration shift to a considerably higher frequency band of $4 \mathrm{~Hz}$, irrespective of the excitation magnitude considered. Such interaction under the side-to-side motion is considerably small due to relatively small biodynamic force developed at the back support driving-point, which is partly caused by lateral sliding of the upper body.

The sitting posture yields small influence on the total power dissipated within the body, derived from the seat pan driving-point measurements, under both axes of vibration of smaller magnitudes $\left(0.25\right.$ and $\left.0.5 \mathrm{~m} / \mathrm{s}^{2}\right)$. An increase in the excitation magnitude to $1 \mathrm{~m} / \mathrm{s}^{2}$, however, caused greater energy transfer for the back supported postures under both axes of vibration. An inclined back support would impose relatively less fore-aft vibration to the upper body compared to vertical support, and help to limit the upper body motion, and thereby result in slightly lower energy transfer. The absorbed power responses under the lateral motion revealed an opposite trend, where the inclined support causes slightly larger power at the back support, which may be attributed to relatively less sliding of the upper body against the inclined back support.

The large variation of the vibration power absorption in different vibration directions and postures suggest that a single frequency weighting is not sufficient for the risk assessment of the horizontal vibration exposure. More specifically, the VPA-based frequency weightings derived in this study suggest that the $W_{d}$-weighting defined in the current ISO-2631-1 is acceptable for assessing the horizontal vibration exposure of human subject seated with- out a back support but it is not appropriate for the sitting posture with the body firmly in contact with the back support. The VPA-based frequency weightings may be used to help develop better frequency weighting for the discomfort and risk assessment.

\section{References}

1) Bovenzi M, Hulshof CTJ (1999) An updated review of epidemiologic studies on the relationship between exposure to whole-body vibration and low back pain (1986-1997). Int Arch Occup Environ Health 72, 351-65.

2) Kumar A, Mahajan P, Mohan D, Vargjese M (2001) Tractor vibration severity and driver health: a study from rural India. J Agric Eng Res 80, 313-28.

3) Marsili A, Ragni L, Vassalini G (1998) Vibration and noise of a tracked forestry vehicle. J Agric Eng Res 70, 295-306.

4) Donati P (1998) A procedure for developing a vibration test method for specific categories of industrial trucks. J Sound Vib 215, 947-57.

5) Bovenzi M, Pinto I, Stacchini N (2002) Low back pain in port machinery operators. J Sound Vib 235, 3-20.

6) Programme for technical risk factors national institute for working life research network on detection and prevention of injuries due to occupational vibration exposures (EU BIOMED 2 concerted Action Programme) 2003. In: Centralized European database whole-body vibration in earth-moving vehicles. http://umetech.niwl. se/vibration/WBVHome.html. Accessed October 13, 2003.

7) Maeda S, Morioka M (1998) Measurement of wholebody vibration exposure from garbage trucks. J Sound Vib 215, 959-64.

8) International Organization for Standardization (1997) Mechanical vibration and shock - evaluation of human exposure to whole-body vibration. Part I: General requirements. International Standard ISO 2631-1.

9) Golding JF, Markley HM, Stott JRR (1994) The effects of motion, body axis, and posture on motion sickness induced by low frequency linear oscillation. Proc. of the 29th UK Group Meeting on Human Response to Vibration, Alverstoke, Gosport (United Kingdom), 19-21 September.

10) Fairley TE, Griffin MJ (1989) The apparent mass of the seated human body: vertical vibration. J Biomech 22, 81-94.

11) Holmlund P, Lundström R, Lindberg L (2000) Mechanical impedance of the human body in the vertical direction. Appl Ergon 31, 415-22.

12) Mansfield NJ, Lundström R (1999) The apparent mass of the human body exposed to non-orthogonal horizontal vibration. J Biomech 32, 1269-78.

13) Nawayseh N, Griffin MJ (2005) Tri-axial forces at the seat and backrest during whole body fore-and-aft vibration. J Sound Vib 281, 921-42.

14) Mandapuram S, Rakheja S, Ma S, Demont R (2005) 
Influence of back support conditions on the apparent mass of seated occupants under horizontal vibration. Ind Health 43, 421-35.

15) Fairley TE, Griffin MJ (1990) The apparent mass of the seated human body in the fore-and-aft and lateral directions. J Sound Vib 139, 299-306.

16) Nawayseh N, Griffin MJ (2005) Non-linear dual-axis biodynamic response to fore-and-aft whole-body vibration. J Sound Vib 282, 831-62.

17) Dong RG, Wu JZ, Welcome DE (2005). Recent advances in biodynamics of hand-arm system. Ind Health 43, 449-71.

18) Lee RA, Pradko F (1968) Analytical analysis of human vibration. SAE Transactions 77, Paper No.680091. Automotive Engineering Congress, Detroit, Michigan, 8-12 January.

19) Burström L (1990) Absorption of vibration energy in the human hand and arm. Ph.D. Thesis in the department of human work sciences, division of physical environment technology, Luleå University of Technology, Sweden.

20) Mansfield NJ, Holmlund P, Lundström R (2001) Apparent mass and absorbed power during exposure to whole-body vibration and repeated shocks. J Sound Vib 248, 427-40.

21) Nawayseh $\mathrm{N}$ (2005) Absorbed power at the seat, backrest and feet of subjects exposed to whole-body vertical vibration. Proc. of the 40th UK Conf. on Human Response to Vibration, Liverpool (United Kingdom), 13-15 September.

22) Wang W, Rakheja S, Boileau P-E (2006), The role of seat geometry and posture on the mechanical energy absorption characteristics of seated occupants under vertical vibration. Int J Ind Ergon 36, 171-84.
23) Lundström R, Holmlund P (1998) Absorption of energy during whole-body vibration exposure. J Sound Vib 215, 789-99.

24) Mansfield NJ, Griffin MJ (1998) Effect of magnitude of vertical whole-body vibration on absorbed power for the seated human body. J Sound Vib 215, 813-25.

25) Lundström R, Holmlund P, Lindberg L (1998) Absorption of energy during vertical whole-body vibration exposure. J Biomech 31, 317-26.

26) Mansfield NJ (2005) Impedance methods (apparent mass, driving point mechanical impedance and absorbed power) for assessment of the biomechanical response of the seated person to whole-body vibration. Ind Health 43, 378-89.

27) Nawayseh N, Griffin MJ (2005) Tri-axial forces at the seat and backrest during whole body vertical vibration. J Sound Vib 281, 309-26.

28) Rakheja S, Stiharu I, Zhang H, Boileau P-E (2006) Seated occupant interactions with seat backrest and pan, and biodynamic responses under vertical vibration. J Sound Vib 298, 651-71.

29) Holmlund P, Lundström R (1998) Mechanical impedance of the human body in the horizontal direction. $\mathrm{J}$ Sound Vib 215, 801-12.

30) Winter DA (1979) Biomechanics of human movement. 151 (Now published as Biomechanics and motor control of human movement. John Wiley \& Sons, Toronto.

31) Dong RG, Welcome DE, McDowell TW, Wu JZ, Schopper AW (2006) Frequency weighting derived from power absorption of fingers-hand-arm system under $\mathrm{z}_{\mathrm{h}}{ }^{-}$ axis. J Biomech 39, 2311-24.

32) Griffin MJ (1990) Handbook of Human Vibration. Academic Press, New York. 